\title{
Sequential task predictability in task switching
}

\author{
IRING KOCH \\ Max Planck Institute for Human Cognitive and Brain Sciences, Munich, Germany
}

\begin{abstract}
Many studies of task switching have found that a prolonged preparation time reduces switch costs. An alternative manipulation of task preparation is based on sequential task predictability, rather than preparation time. In Experiments 1 and 2 of the present study, participants performed explicitly instructed task sequences (i.e., AABB) and were then transferred to a random sequence. The observed benefit of predictability-based task preparation was not switch specific. In Experiment 3, the participants changed from random to predictable tasks. The observed predictability benefit again was not switch specific. The data thus suggest that task switching does not necessarily require a switch-specific reconfiguration process. Rather, task-specific control processes may be needed in both task switches and repetitions.
\end{abstract}

A vital question in cognitive psychology concerns the mechanisms of action control for changing task requirements. This control function has been ascribed to the configuration of cognitive processes, also termed task set. Recently, the dynamics of task set have been studied using task switching. In task-switching experiments, performance when participants switch between tasks is compared with that when the task is repeated. Typically, reaction time (RT) is longer with switches than with repetitions, indicating switch costs (see Monsell, 2003, for a review).

The goal of the present study was to explore task preparation processes in task switching. Many studies have shown that having a long preparation interval reduced RTs in task switches more than in repetitions, thus reducing switch costs. This effect of preparation on switch costs has been attributed to a task set reconfiguration process (e.g., Meiran, 1996; Rogers \& Monsell, 1995). According to reconfiguration accounts, reconfiguration is required only in a task switch, but not in a task repetition, so that preparation benefits should be predicted to have a substantial switch-specific component.

However, the results of several studies of task preparation do not conform to this prediction. In some of these studies, preparation has been manipulated by varying task predictability, using an incidental sequence-learning methodology, instead of providing either a long or a short preparation time (Gotler, Meiran, \& Tzelgov, 2003; Heuer, Schmidtke, \& Kleinsorge, 2001; Koch, 2001). For instance, in Gotler et al.'s study, participants performed an eight-trial sequence of two different tasks. The participants were not informed about the predictable sequence, and each task was indicated by an instruction cue. Gotler

Thanks go to Miriam Gade, Peter Keller, Andrea Philipp, and Stefanie Schuch for discussions and to Nachshon Meiran, Tram Neill, and Eric Ruthruff for helpful comments. Correspondence concerning this article should be addressed to I. Koch, Max Planck Institute for Human Cognitive and Brain Sciences, Department of Psychology, Amalienstrasse 33, D-80799 Munich, Germany (e-mail: iring.koch@cbs.mpg.de). et al. found an increased RT when participants were transferred to random tasks, indicating that the predictable sequence, at least partially, had been learned. Importantly, as in previous studies (Heuer et al., 2001; Koch, 2001), the preparation benefit was the same for switches and repetitions, so that it did not affect the size of the switch costs, unlike manipulations of preparation time.

Furthermore, other studies in which quite different experimental manipulations have been used to induce task predictability have reported the same surprising equivalence of the predictability benefit for task switches and repetitions. For example, Sohn and Carlson (2000) had participants perform trials consisting of pairs of tasks. Each task was unambiguously cued by the color of the bivalent stimuli, but for half of the participants it was also the case that the task transition was predictable. Predictability produced a large performance benefit relative to unpredictable tasks, and this benefit was equal for switches and repetitions. Likewise, Ruthruff, Remington, and Johnston (2001) induced task expectancy by presenting univalent stimuli, which unambiguously cue only one task, in a predictable sequence and occasionally introducing violations of the sequence. These violations were equally harmful for switches and repetitions. That is, these two studies reported the same data pattern as incidental task sequence learning studies (see also Dreisbach, Haider, \& Kluwe, 2002).

Together, these observations of the switch-unspecific benefits of predictability apparently represent a challenge for accounts proposing that preparation benefits in task switching are due to an advance preparation process that is specific for a task switch (e.g., Meiran, 1996; Rogers \& Monsell, 1995; Rubinstein, Meyer, \& Evans, 2001).

However, in view of these challenging observations, one might object that methodological factors may have precluded a switch-specific preparation benefit in these studies. For instance, incidental learning studies did not explicitly instruct participants to use the task sequence for task preparation. It thus remained to be tested whether 
explicit instructions to use the sequence would yield switch-specific preparation effects. Furthermore, in Sohn and Carlson's (2000) study, the manipulation of predictability was between subjects. Recently, it has been shown that between-subjects manipulations of task preparation are much less effective than within-subjects manipulations (cf. Altmann, 2004; Koch, 2001). Therefore, Sohn and Carlson's study might have underestimated the potential switch-specific preparation benefit, even though the benefit they observed was quite substantial. Finally, Ruthruff et al. (2001) used univalent stimuli. Such stimuli result in rather small switch costs, whereas switch costs are typically much larger with bivalent stimuli, which are supposed to increase stimulus-triggered task conflict (Rogers \& Monsell, 1995). Furthermore, due to the violations of predictability, participants always had to await stimulus onset prior to the final task decision, which might have reduced the predictability benefit. Hence, the predictability benefit might have been underestimated also in Ruthruff et al.'s study.

The goal of the present three experiments was to examine whether the benefit of sequential task predictability would be switch specific when the task sequence was explicitly instructed, short, and easy to remember (AABB; cf. Rogers \& Monsell, 1995). Moreover, as in studies on incidental task sequence learning, a within-subjects negative transfer design was used, so that a lack of a switchspecific preparation benefit, if found, could not be attributable to the use of a between-subjects design (cf. Altmann, 2004; Koch, 2001), as in Sohn and Carlson's (2000) study. Finally, bivalent stimuli were used. It is known that the effects of preparation interval are much larger with bivalent stimuli than with univalent stimuli (Rogers \& Monsell, 1995), suggesting that the predictability benefit would be larger than that found by Ruthruff et al. (2001) with univalent stimuli.

In the experiments, the participants were explicitly encouraged to use the task sequence for preparation. The participants were also provided with a redundant task cue. Note that cue-based preparation is possible in both predictable and random tasks but that effects of sequential task predictability can be present only in predictable tasks. Hence, any predictability benefits should be lost in random sequences, and the size of this loss would provide a measure of the predictability effect (Gotler et al., 2003; Heuer et al., 2001; Koch, 2001).

\section{EXPERIMENT 1}

In Experiment 1, the response-stimulus interval (RSI) was $1,000 \mathrm{msec}$. Preparation intervals of this length are known to lead to robust task preparation in predictable sequences (Rogers \& Monsell, 1995). The explicit task cue preceded the stimulus by $900 \mathrm{msec}$. The crucial question was whether explicitly instructed task predictability would affect switch costs.

\section{Method}

Participants. Sixteen participants took part and received $5 €$.
Stimuli and Tasks. The stimuli were the digits $1-9$, excluding 5 . The digits were $0.8 \mathrm{~cm}$ high, centrally presented inside a square or diamond frame measuring $3.8 \times 3.8 \mathrm{~cm}$ on a 15 -in. screen. Stimulus presentation and response registration were synchronized with the refresh rate of the screen. The tasks were to decide whether the digit was odd or even or whether it was smaller or greater than 5 . Stimulus order was random, but immediate repetition was not allowed. Responses were made on either the left or the right button of an external response panel. Response keys measured $1.7 \times 1.7 \mathrm{~cm}$, separated by $3.3 \mathrm{~cm}$.

Procedure. The participants were informed that each of the stimuli would be presented in a frame, that responses should be fast but accurate, and that they would have to perform fixed task sequences of 12 trials: AABBAABBAABB. Whether $A$ denoted the odd/even or the greater/smaller task was held constant for each participant but was counterbalanced across participants. A schema of the stimulus-response (S-R) mappings for each task was placed beneath the screen. S-R mapping was counterbalanced across participants. Following incorrect keypresses, an error message appeared for $500 \mathrm{msec}$ at the bottom of the screen.

Before each run of 12 trials, a message emphasized using the interval prior to the next stimulus for task preparation. The participants were then told that the shape of the stimulus presentation frame (square vs. diamond) would covary with the task to provide an external, redundant cue. To accomplish this, each response caused the frame to disappear and reappear on the screen after $100 \mathrm{msec}$ in the shape associated with the next task. The cue-stimulus interval (CSI) was $900 \mathrm{msec}$.

After each run of 12 trials, the participants received feedback of their mean RT and were encouraged to improve performance. The participants started with 8 practice trials. The participants then went through seven blocks of four runs each. In Blocks 1-5 and 7, the predictable 4-trial task sequence was presented. In Block 6 , the task sequence was randomized (cf. Heuer et al., 2001), with the constraint that each task still appeared equally frequently and that a given task could not appear more than three times in a row. Prior to each run in Block 6, a message on the screen indicated that a nonpredictable task sequence would occur. At the start of Block 7, a message indicated the reintroduction of the predictable task sequence. The experiment took about $30 \mathrm{~min}$.

Design. The independent variables were task predictability (predictable vs. random sequence) and trial type (switch vs. repetition) as within-subjects variables. The main dependent variable was RT. The measure of task predictability was calculated by averaging performance in Blocks 5 and 7 and subtracting this value from that for Block 6. (Block 7 was included to correct this measure for unspecific practice effects.) Significance tests were conducted with an alpha of .05.

\section{Results and Discussion}

The first two trials of each run were discarded as warmup trials. For the RT analysis, incorrect trials, those trials following them, and RTs above 3,000 msec were discarded (outliers; fewer than 1\%). Then the mean was determined for each participant as a function of trial type and block. The data of one extremely slow participant (mean RT averaged across all blocks was $1,379 \mathrm{msec}$, as compared with a mean of $708 \mathrm{msec}$ for the remaining participants) were not analyzed.

Figure 1 shows the RT data as a function of trial type and block. First, the data of the Pretransfer Blocks 1-5 were submitted to an analysis of variance (ANOVA) with trial type and block as variables. This ANOVA yielded significant effects of trial type $\left[F(1,14)=25.174, M S_{\mathrm{e}}=\right.$ $70,689]$ and block $\left[F(4,56)=4.536, M S_{\mathrm{e}}=12,685\right]$. Both 


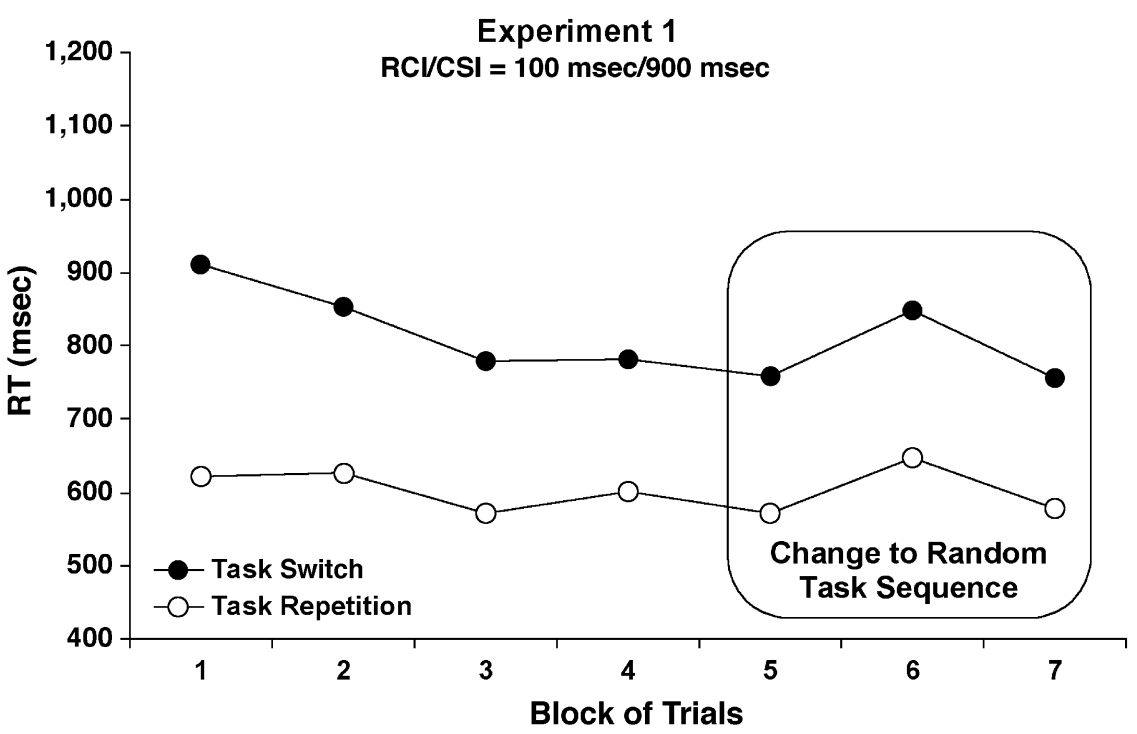

Figure 1. Experiment 1: mean reaction time (RT, in milliseconds) as a function of block of trials and trial type (switch vs. repetition). There was a predictable task sequence in Blocks 1-5 and 7 and a random task sequence in Block 6. RCI, response-cue interval; CSI, cuestimulus interval.

variables interacted $\left[F(4,56)=2.614, M S_{\mathrm{e}}=13,939\right]$, showing that switch costs were reduced with practice.

To test the effect of task predictability, RTs for the predictable sequence Blocks 5 and 7 were collapsed and then compared with the data for Block 6. An ANOVA with predictability and trial type as variables revealed significant effects of trial type $\left[F(1,14)=23.143, M S_{\mathrm{e}}=\right.$ 24,051], showing switch costs of $193 \mathrm{msec}$, and predictability $\left[F(1,14)=6.649, M S_{\mathrm{e}}=15,022\right]$, indicating that RT increased by $81 \mathrm{msec}$ when predictability was removed in Block 6. However, there was no significant interaction $(F<1, p=.716)$, showing that switch costs were not significantly larger in random tasks $(201 \mathrm{msec})$ than in predictable tasks $(184 \mathrm{msec})$.

Error rates were submitted to the same analysis as RTs. Error rate increased in random tasks $(7.3 \%$ vs. $5.3 \%)$, and it was larger in switch trials (6.9\% vs. $5.6 \%)$, but no effect was significant.

In sum, the data replicate the earlier finding that the benefit of task predictability is not specific to task switches (Dreisbach et al., 2002; Gotler et al., 2003; Heuer et al., 2001; Koch, 2001; Ruthruff et al., 2001; Sohn \& Carlson, 2000).

However, in Experiment 1, the change in predictability was accompanied by a change in task instruction, which could lead by itself to some kind of restart costs (cf. Allport \& Wylie, 2000). Importantly though, in Experiment 1, the RT increase in Block 6 was not accompanied by decreased error rates, so there was no speedaccuracy tradeoff due to more cautious responding after a change in instructions. Moreover, the switch back to predictable tasks in Block 7 was also associated with a change in instructions, but performance returned to the level of Block 5. Nevertheless, Experiments 2 and 3 were run to confirm that the present effect was due to task predictability, rather than to restart costs.

\section{EXPERIMENT 2}

In Experiment 1, the participants presumably relied to some degree on the cue because the CSI was rather long, even when sequential predictability was available. In Experiment 2, the CSI was reduced to $100 \mathrm{msec}$ to increase the predictability benefit, relative to exclusively cuebased performance in random tasks. If the transfer effect found in Experiment 1 had been entirely due to restart costs, the transfer effect should be unchanged by the CSI manipulation. If, however, predictability is important, the transfer effect should be enlarged in Experiment 2.

\section{Method}

Participants. Sixteen participants took part and received $5 €$.

Stimuli, Procedure, and Design. The stimuli, procedure, and design were like those in Experiment 1, except that the CSI was set to $100 \mathrm{msec}$ and the response-cue interval to $900 \mathrm{msec}$.

\section{Results and Discussion}

There were fewer than $1 \%$ outliers (i.e., RT $>3,000$ msec). For the RT data (see Figure 2), the ANOVA for Blocks $1-5$ yielded an effect of trial type $[F(1,15)=$ $\left.57.163, M S_{\mathrm{e}}=32,652\right]$ and block $[F(4,60)=18.883$, $\left.M S_{\mathrm{e}}=17,168\right]$. The interaction was not significant $(p>$ $.14)$.

For the transfer test, there were significant effects of predictability $\left[F(1,15)=24.042, M S_{\mathrm{e}}=33,719\right]$, indicating a predictability effect of $225 \mathrm{msec}$, and trial type 


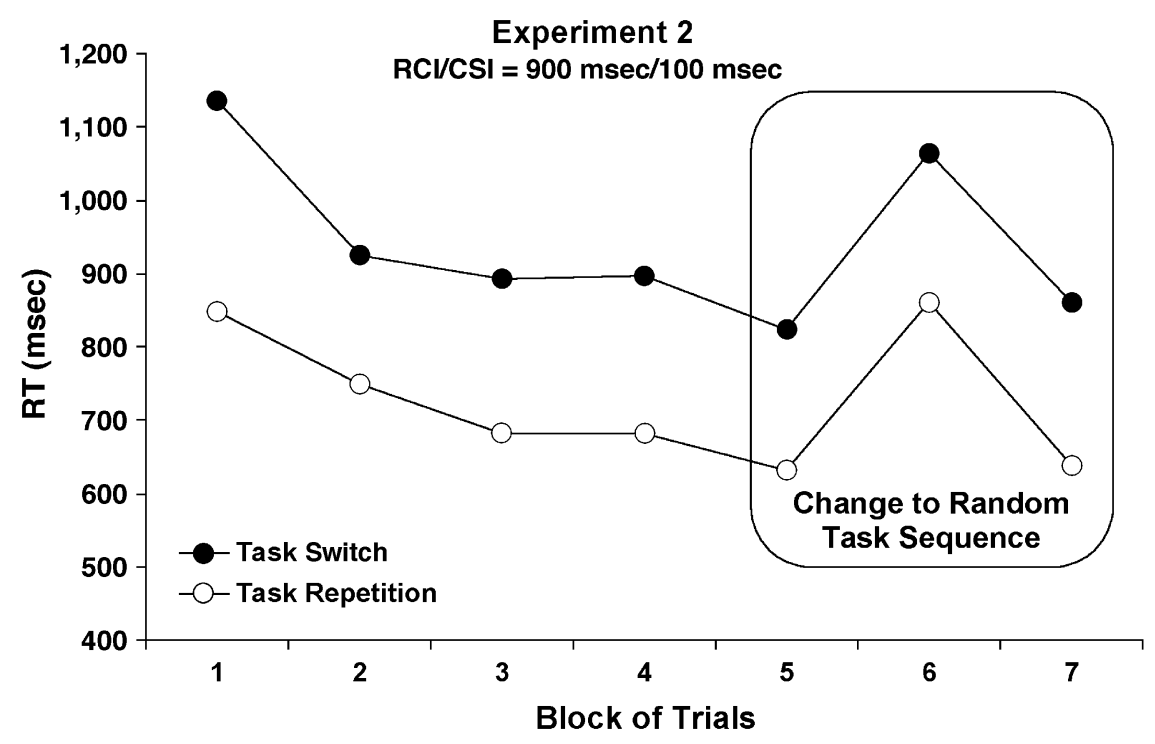

Figure 2. Experiment 2: mean reaction time (RT, in milliseconds) as a function of block of trials and trial type (switch vs. repetition). There was a predictable task sequence in Blocks 1-5 and 7 and a random task sequence in Block 6. RCI, response-cue interval; CSI, cuestimulus interval.

$\left[F(1,15)=82.354, M S_{\mathrm{e}}=8,160\right]$. Switch costs were $203 \mathrm{msec}$ in random tasks and $207 \mathrm{msec}$ in predictable tasks ( $p=.917$, for the interaction), replicating the pattern observed in Experiment 1.

Importantly, when tested in a between-experiment ANOVA, the present 225-msec transfer effect was significantly larger than the 81-msec effect in Experiment 1 $\left[F(1,29)=6.456, M S_{\mathrm{e}}=24,693\right]$. This increase of the negative transfer effect would be difficult to explain by the restart account but is fully in line with the effect of predictability.

Note that the long cue-based preparation interval in Experiment 1, relative to that in Experiment 2, decreased both RT in general [ 707 vs. $850 \mathrm{msec} ; F(1,29)=7.240$, $\left.M S_{\mathrm{e}}=87,698\right]$ and, most important, the predictability benefit ( 81 vs. $225 \mathrm{msec}$; see above), but it did not significantly decrease the size of switch costs (193 vs. $205 \mathrm{msec}$; $F=0.074, p=.787)$. This observation of general preparation benefits but uninfluenced switch costs when a between-subjects manipulation of preparation time was used replicates earlier findings (Altmann, 2004; Koch, 2001). However, the present manipulation of predictability was made within subjects, so that the lack of a switchspecific benefit cannot be attributed to design factors.

In error rates, only the interaction was significant $\left[F(1,15)=5.698, M S_{\mathrm{e}}=0.0021\right]$. Switch costs were smaller in random tasks (1.4\%) than in predictable tasks (4.1\%), due to a decrease of error rate in switches and an increase in repetitions, which is quite inexplicable.

\section{EXPERIMENT 3}

To further advance the understanding of the predictability effect and to completely rule out the restart account of the transfer effect, the change in predictability in Experiment 3 was from random to predictable tasks, rather than vice versa. This change should lead to a benefit with the change in instructions, whereas the restart account would predict a cost.

\section{Method}

Participants. Sixteen participants took part and received $5 €$. Stimuli, Procedure, and Design. The only difference from Experiment 2 was that the task sequence was random throughout, except for Block 6, where it was predictable.

\section{Results and Discussion}

There were $0.61 \%$ outliers (RT $>3,000 \mathrm{msec}$ ). For the RT data (see Figure 3), the ANOVA for Blocks 1-5 yielded an effect of trial type $\left[F(1,15)=15.044, M S_{\mathrm{e}}=24,154\right]$ and block $\left[F(4,60)=6.653, M S_{\mathrm{e}}=23,558\right]$. The interaction was not significant $(F<1)$.

For the transfer test, there were significant effects of trial type $\left[F(1,15)=52.651, M S_{\mathrm{e}}=9,295\right]$ and predictability $\left[F(1,15)=6.735, M S_{\mathrm{e}}=21,801\right]$. The effect of predictability was due to a 96-msec decrease of RT when predictability was introduced, but this effect interacted with trial type $\left[F(1,15)=11.178, M S_{\mathrm{e}}=11,176\right]$. For switches, this decrease was only $7 \mathrm{msec}$, whereas it was $184 \mathrm{msec}$ for repetitions, so that switch costs were $86 \mathrm{msec}$ in random tasks and increased to $263 \mathrm{msec}$ in predictable tasks. (For error rates, all the effects were not significant; $F_{\mathrm{s}}<1$.)

In summary, Experiment 3 showed that the change in instructions had a positive, rather than a negative, effect on performance, which argues against a restart cost account and is in line with the notion that predictability is crucial for explaining the results.

Interestingly, switch costs in random tasks were only $96 \mathrm{msec}$ (averaged over Blocks 1-5), whereas the corre- 


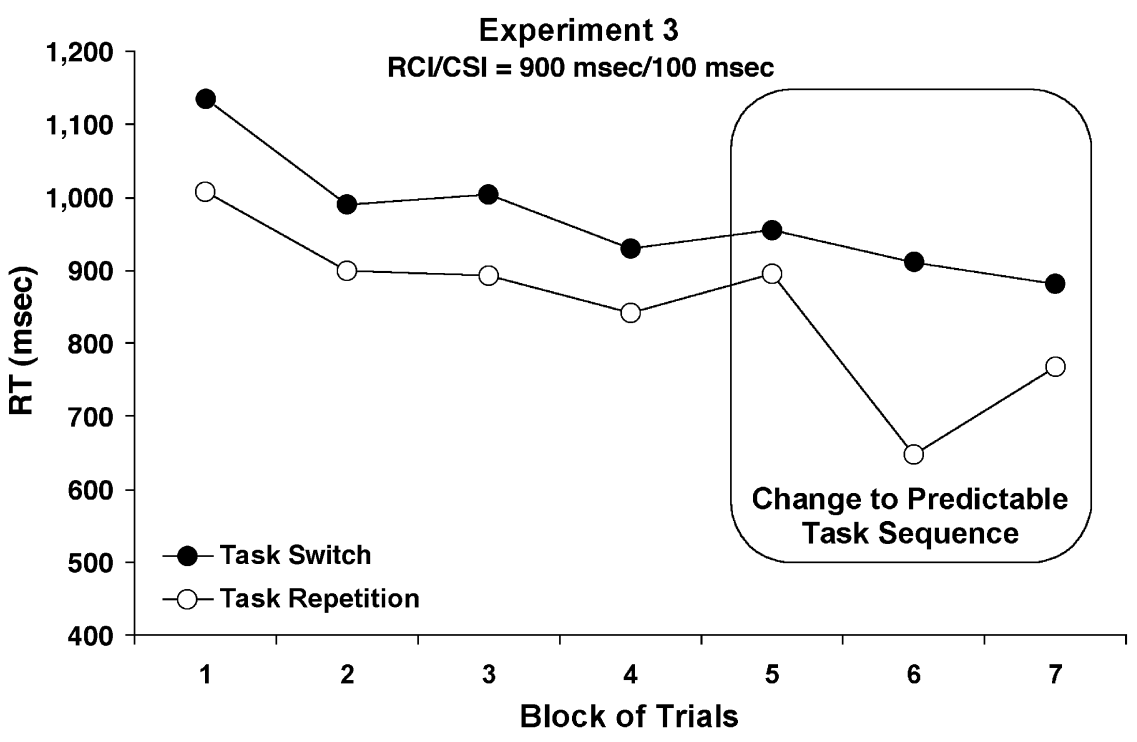

Figure 3. Experiment 1: mean reaction time (RT, in milliseconds) as a function of block of trials and trial type (switch vs. repetition). There was a random task sequence in Blocks 1-5 and 7 and a predictable task sequence in Block 6. RCI, response-cue interval; CSI, cuestimulus interval.

sponding cost with predictable tasks in Experiment 2 was $216 \mathrm{msec}$. This difference was significant when tested between experiments $\left[F(1,30)=10.258, M S_{\mathrm{e}}=\right.$ 28,403; see the General Discussion section].

\section{GENERAL DISCUSSION}

The present experiments addressed the question of whether sequential task predictability would affect switch costs. In Experiments 1 and 2, substantial predictability benefits were found, and these were equal for task switches and repetitions. The predictability benefit in these experiments appears to have been greater than that reported in incidental learning studies (Gotler et al., 2003; Heuer et al., 2001; Koch, 2001), but this difference in the size of the predictability benefit is most likely due to the combination of using explicit instructions and a shorter task sequence, which makes a formal comparison across studies difficult.

The finding that predictability-based task preparation is not switch specific is also consistent with findings observed with other manipulations of predictability (Dreisbach et al., 2002; Ruthruff et al., 2001; Sohn \& Carlson, 2000). Importantly, the present experiments, by using explicit sequence instructions, bivalent stimuli, and a within-subjects manipulation of predictability, were able to rule out several potential objections against the interpretation of earlier findings. Thus, the data clearly show that task preparation effects due to sequential predictability are not switch specific, even when they are substantial, such as the 225-msec benefit in Experiment 2.

Finding no switch-specific preparation benefits but, at the same time, substantial preparation benefits in task repetitions is not readily explained by reconfiguration accounts. Rather, this data pattern suggests that a kind of goal-setting or task-updating process is equally needed in task switches and repetitions (for a discussion, see also Fagot, 1994; Gotler et al., 2003; Ruthruff et al., 2001).

The data in Experiment 3 reinforce the conclusion that predictability-based task preparation benefits are not switch specific. Experiment 3 was conducted to demonstrate that a transfer from random to predictable tasks improves performance, due to the added benefit of sequential predictability. This improvement indeed occurred. However, the predictability benefit in Experiment 3 was stronger for repetitions than for switches, thus even increasing switch costs (or repetition benefits). Although this preparation-based increase of switch costs is certainly inconsistent with the idea that preparation triggered an extra reconfiguration process that occurs only in a task switch, it is also puzzling in light of the alternative view that there is a task-updating (or goalsetting) process required in both task switches and repetitions. Why should this process have stronger effects in repetitions than in switches?

It appears that the puzzling increase of switch costs is primarily due to the relatively small switch costs in random tasks, and when tested between experiments, the switch costs were indeed significantly smaller than those in Experiment 2. Presently, though, the meaning of this finding is unclear, because it is inconsistent with Sohn and Carlson's (2000) data, who observed equal switch costs in predictable and random tasks in a between-subjects comparison. However, Tornay and Milàn (2001, Experiment 3) observed (also between subjects) slightly but significantly higher switch costs in predictable tasks (but see Monsell, Sumner, \& Waters, 2003, for a discussion), which is not consistent with the present Experiments 1 and 
2 and with the other studies on task predictability reviewed above. Thus, for the time being, it seems prudent not to put too much emphasis on the finding of Experiment 3 that switch costs are smaller with random than with predictable tasks, even though it seems to be important to further explore this finding in future research. However, this puzzling aspect of Experiment 3 should not detract from the main result of all the experiments in the present article, which is the demonstration that predictabilitybased task preparation benefits are not switch specific.

In summary, finding switch-specific preparation benefits with prolonged preparation time is usually taken as the major argument for proposing a process of task set reconfiguration that is required only in a task switch (see Monsell, 2003), but studies on preparation due to predictability apparently do not support this proposal. Rather, these studies suggest a general task-updating control process (see also Ruthruff et al., 2001; Sohn \& Carlson, 2000). According to this alternative account, the chain of task-specific processes is not necessarily qualitatively different in a task switch and a task repetition, and the switch cost is rather based on different durations of the component processes, such as task updating or response selection (Allport \& Wylie, 2000; Schuch \& Koch, 2003).

Thus, the effects of predictability do not seem to warrant a switch-specific reconfiguration process. There are also recent studies that have questioned the interpretation of the switch-specific benefit with prolonged preparation time (Dreisbach et al., 2002; Logan \& Bundesen, 2003) or its generality (Altmann, 2004). It thus appears that the theoretical focus on a switch-specific reconfiguration process has greatly inspired empirical research on control processes in task switching but that the results of this research are still consistent with the idea that the hypothesized control process occurs also in task repetitions. It remains to be determined when exactly the contribution of this control process is larger in task switches than in repetitions.

\section{REFERENCES}

Allport, A., \& Wylie, G. (2000). Selection-for-action in competing (Stroop) tasks: "Task-switching," stimulus-response bindings, and negative priming. In S. Monsell \& J. S. Driver (Eds.), Attention and performance XVIII: Control of cognitive processes (pp. 35-70). Cambridge, MA: MIT Press.
Altmann, E. M. (2004). The preparation effect in task switching: Carryover of SOA. Memory \& Cognition, 32, 153-163.

Dreisbach, G., Haider, H., \& Kluwe, R. H. (2002). Preparatory processes in the task-switching paradigm: Evidence from the use of probability cues. Journal of Experimental Psychology: Learning, Memory, \& Cognition, 28, 468-483.

FAGOT, C. (1994). Chronometric investigations of task switching. Unpublished doctoral thesis, University of California, San Diego.

Gotler, A., Meiran, N., \& Tzelgov, J. (2003). Nonintentional task set activation: Evidence from implicit task sequence learning. Psychonomic Bulletin \& Review, 10, 890-896.

Heuer, H., SchmidtKe, V., \& KLEINSORGe, T. (2001). Implicit learning of sequences of tasks. Journal of Experimental Psychology: Learning, Memory, \& Cognition, 27, 967-983.

KoCH, I. (2001). Automatic and intentional activation of task sets. Journal of Experimental Psychology: Learning, Memory, \& Cognition, 27, 1474-1486.

LOGAN, G. D., \& Bundesen, C. (2003). Clever homunculus: Is there an endogenous act of control in the explicit task cuing procedure? Journal of Experimental Psychology: Human Perception \& Performance, 29, 575-599.

MEIRAN, N. (1996). Reconfiguration of processing mode prior to task performance. Journal of Experimental Psychology: Learning, Memory, \& Cognition, 22, 1423-1442.

Monsell, S. (2003). Task switching. Trends in Cognitive Sciences, 7, 134-140.

Monsell, S., Sumner, P., \& Waters, H. (2003). Task-set reconfiguration with predictable and unpredictable task switches. Memory \& Cognition, 31, 327-342.

RoGERS, R. D., \& MONSELL, S. (1995). Costs of a predictable switch between simple cognitive tasks. Journal of Experimental Psychology: General, 124, 207-231.

Rubinstein, J., Meyer, D. E., \& Evans, J. E. (2001). Executive control of cognitive processes in task switching. Journal of Experimental Psychology: Human Perception \& Performance, 27, 763-797.

RuthrufF, E., Remington, R. W., \& Johnston, J. C. (2001). Switching between simple cognitive tasks: The interaction of top-down and bottom-up factors. Journal of Experimental Psychology: Human Perception \& Performance, 27, 1404-1419.

ScHUCH, S., \& KoCH, I. (2003). The role of response selection for inhibition of task sets in task shifting. Journal of Experimental Psychology: Human Perception \& Performance, 29, 92-105.

SoHn, M.-H., \& CARLSON, R. A. (2000). Effects of repetition and foreknowledge in task-set reconfiguration. Journal of Experimental Psychology: Learning, Memory, \& Cognition, 26, 1445-1460.

ToRnAY, F. J., \& MiLÀn, E. G. (2001). A more complete task-set reconfiguration in random than in predictable task switch. Quarterly Journal of Experimental Psychology, 54A, 785-803.

(Manuscript received December 20, 2002; revision accepted for publication January 30, 2004.) 